\title{
Multiscale Numerical Models for Simulation of Radiation Events in Semiconductor Devices
}

\author{
Alexander I. Fedoseyev ${ }^{1}$, Marek Turowski ${ }^{1}$, Ashok Raman ${ }^{1}$, Michael L. Alles ${ }^{2}$, \\ and Robert A. Weller ${ }^{2}$ \\ ${ }^{1}$ CFD Research Corporation (CFDRC), 215 Wynn Drive, Huntsville, AL 35805 \\ aifacfarc.com \\ ${ }^{2}$ Vanderbilt University, Nashville, Tennessee 37237, USA
}

\begin{abstract}
This paper describes the new CFDRC mixed-mode simulator which combines multiscale 3D Technology Computer Aided Design (TCAD) device models (fluid carrier transport and nuclear ion track impact), and advanced compact transistors models. Key features include an interface and 3D adaptive meshing to allow simulations of single event radiation effects with nuclear reactions and secondary particles computed by Vanderbilt's MRED/Geant4 tools.
\end{abstract}

Keywords: Nanoscale device, radiation event, nuclear reaction, 3D transient simulation, 3D adaptive mesh generation, hydrodynamics, drift-diffusion, multiscale, finite volume method, computer-aided-design.

\section{Introduction}

Space and ground-level electronic equipment with devices of nanometer-scale, extremely high densities and highly complex circuits may be strongly affected by ionizing radiation. Accurate multiscale numerical models are necessary for better analysis of radiation effects and design of radiation tolerant devices [1,2]. Nano-scale electronic device responses to radiation are strongly related to the microstructure of the radiation event. Multiscale models and adaptive dynamic 3D mesh generation are needed to enable efficient simulations of transient semiconductor device and circuit responses to realistic multi-branched track, produced by ionizing particle. Predictive calculations can leverage efficient mixed-mode simulation capability, that is, threedimensional (3D) physical simulations of ionizing radiation effects in semiconductor structure coupled with external circuit solver. To design radiation-hardened (rad-hard) electronics and predict circuit/system characteristics, modeling tools are required at multiple levels (Fig. 1).

The energy deposition by ionizing particles (so called single event effects) can no longer be treated as an average deposition (linear energy transfer, LET) along a linear path (Fig. 2). The impact of such radiation events has implications for nano-scale devices reliability operating in space exploration environments. The new multiscale approach to understanding the single-event response of semiconductor materials, devices, and circuits is necessary for reliable engineering models. 
Adaptive dynamic 3D mesh generation capability has been developed to allow complex, multi-branched track data generated by Vanderbilt's Geant4/MRED tools [1,2] simulations to be incorporated into CFDRC NanoTCAD device simulator [3-5] for transient device response simulations (Fig 3).

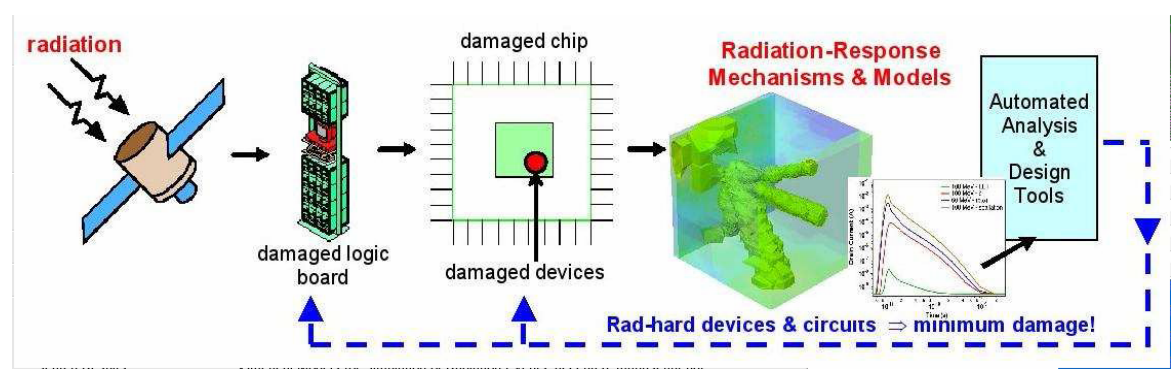

Fig. 1. Space electronics systems require automated design tools across multiple levels: macro, micro, nano. More accurate radiation-effects models are needed for new, nano-scale generations of semiconductor technologies, devices, and ICs.

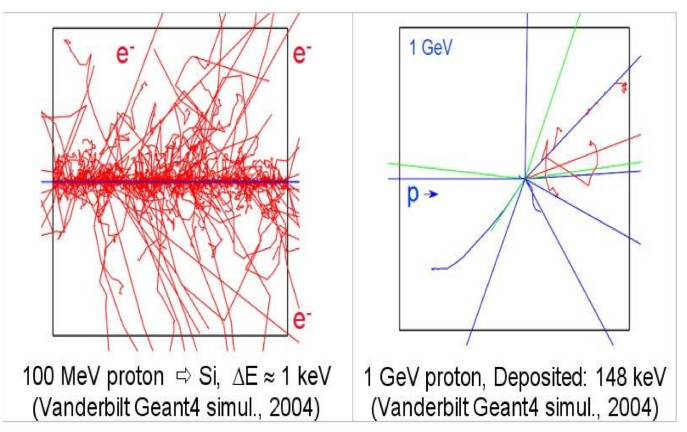

Fig. 2. Examples of realistic ion tracks, to be simulated for device respond, computed by the Vanderbilt University group (middle: $100 \mathrm{MeV}$ proton of energy goes through strikes silicon device, generates hundreds of secondary tracks; right: $1 \mathrm{GeV}$ proton strike generates few secondary high-energy tracks)

In the following sections we provide the description of the multiscale simulation approach with focus on the new adaptive 3D mesh generation around ion tracks, and efficient solution methods for large 3D unstructured meshes which are necessary for transient radiation effects simulations.

\section{Numerical Models Implemented and Governing Equations}

The numerical models for efficient 3D simulation of realistic radiation events in semiconductor nano-scale devices are being integrated within the advanced software tool NanoTCAD, which is a $3 \mathrm{D}$ device simulator developed and commercialized by 
(a)

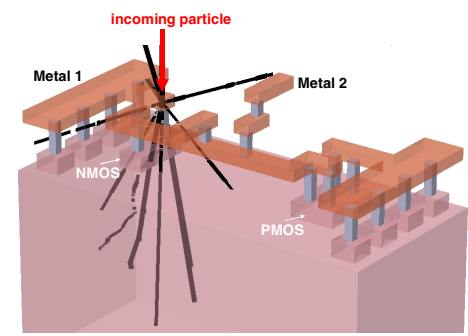

(b)

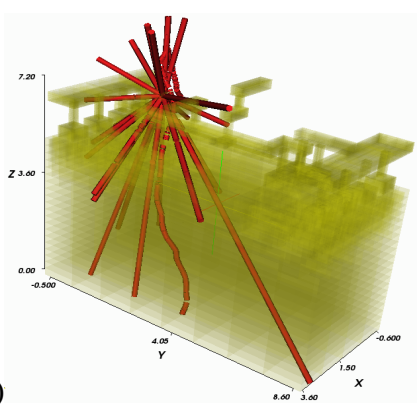

Fig. 3. (a) 3D model of the CMOS inverter test structure, with two metal layers (M1 and M2) and examples of two radiation events computed by MRED/Geant4, where the incoming particle is hitting M1 (copper) above the NMOS-source tungsten contact. (b) 3D mesh generated (semitransparent) for NanoTCAD simulator with ion tracks shown.

CFD Research Corporation [4]. This integration provides a user-friendly interface and a large database of the semiconductor material properties available in NanoTCAD. It also makes possible a complete PV-cell simulation including both quantum and classical models for the appropriate PV-cell elements, both DC and transient regimes, etc.

The models are currently being extended to incorporate simulation of the electronphonon transport in QDS made of semiconductors with both cubic and hexagonal crystal lattice, e.g., InAs/GaAs, $\mathrm{Ge} / \mathrm{Si}, \mathrm{CdSe}, \mathrm{ZnO}$. The drift-diffusion model implemented in NanoTCAD is described below.

\subsection{Fluid Carrier Transport Model}

Fluid carrier transport drift-diffusion models are formulated based on continuity equations for electrons and holes and Poisson equation for electrostatic potential. They are able to provide good comparison with experimental data for transistors with channel length down to $15 \mathrm{~nm}$.

Conservation of charge for electrons is represented by continuity equation

$$
q \frac{\partial n}{\partial t}+\nabla \cdot J_{n}=q R
$$

and similarly for holes as

$$
q \frac{\partial p}{\partial t}+\nabla \cdot J p=-q R
$$

where the electron current is

$$
J_{n}=q \mu_{n}\left(U_{T} \nabla n-n \nabla \Psi\right)
$$

and hole current is

$$
J_{p}=-q \mu_{n}\left(U_{T} \nabla p-p \nabla \Psi\right)
$$


Here $n$ and $p$ are electron and hole densities $\left[1 / \mathrm{cm}^{3}\right], \Psi$ is electrostatic potential [V], $q$ is carrier charge (electron charge $e$ ), $U_{T}=\frac{k_{B} T}{q}$, and diffusion coefficients are $D_{n}=U_{T} \mu_{n}$ and $D_{p}=U_{T} \mu_{p}\left[\mathrm{~cm}^{2} / \mathrm{s}\right]$.

Electron and hole mobilities $\mu_{n}, \mu_{n}$ are calculated parameters (models depend on the material, device, or calculated from quantum or kinetic level problems).

Electrostatic potential which appears in current equations is governed by Poisson equation

$$
\nabla \varepsilon \nabla \Psi=q(n-p-C)
$$

where $\Psi$ is electrostatic potential $[\mathrm{V}], \varepsilon$ is dielectric constant, and $C$ is a doping, $C=N_{D^{-}}^{+}-N_{A}^{-}$where $N_{D}^{+}$and $N_{A}^{-}$are constitutive doping.

\subsection{Boundary Conditions}

Boundary conditions for $n, p$, and $\Psi$ are shown below for the example of Ohmic contact. At the Ohmic contact we assume thermal equilibrium and vanishing space charge which results in

$$
\begin{aligned}
& n \cdot p-n_{i}^{2}=0 \\
& n-p-C=0
\end{aligned}
$$

Solving a quadratic equation for $n, p$ we get Dirichlet conditions for $n$ and $p$ on the boundary (Ohmic contact)

$$
\begin{aligned}
& n_{0}=\frac{1}{2}\left(\sqrt{C^{2}+4 n_{i}^{2}}+C\right) \\
& p_{0}=\frac{1}{2}\left(\sqrt{C^{2}+4 n_{i}^{2}}-C\right)
\end{aligned}
$$

The boundary potential at an Ohmic contact is the sum of the externally applied potential (voltage) $V_{C}(t)$ and the so called built-in potential, which is produced by doping

$$
\Psi=\Psi_{b i}+V_{C}(t)
$$

The built-in potential is 


$$
\Psi_{b i}=U_{T} \ln \left[\frac{C(x)+\sqrt{C^{2}+4 n_{i}^{2}}}{2 n_{i}}\right]
$$

where the intrinsic concentration $n_{i}$ is:

$$
n_{i}=\sqrt{n \cdot p}
$$

\subsection{Discretization of Governing Equations and Efficient Solution of Linear Algebraic System}

Governing equations (1) to (4), (5) are discretized by finite volume method and solved simultaneously using the Newton technique, to ensure a good convergence. Unstructured adaptive meshes are used for domain discretization. In NanoTCAD, we use a high-performance iterative linear solver CNSPACK, developed by Fedoseyev et al. in (2001) [8]. CNSPACK uses a high order preconditioning by incomplete decomposition to ensure the accuracy, stability and convergence of the simulations.

The linear algebraic system is solved in CNSPACK using a CGS-type iterative method with preconditioning by the incomplete decomposition of the matrix. Comparing the CGS and GMRES methods in different tests, it was found that both methods converge well, if a good preconditioner is used. The CGS method needs less memory to store only eight work vectors.

To reduce the memory requirements, a compact storage scheme for matrices is used in CNSPACK. It stores only the nonzero matrix entries. The incomplete decomposition $(I D)$ used for preconditioning, is constructed as a product of triangular and diagonal matrices, $P=L D U$. To avoid diagonal pivot degeneration, the Kershaw diagonal modification is used [9]. If the value of diagonal element becomes small during the construction of preconditioning matrix, i.e. $\left|a_{i i}\right|<\alpha=\sqrt{2^{-t} \sigma \mu}$, the diagonal $a_{i i}$ is replaced by $\alpha$. Here $\sigma$ and $\mu$ are the maximum magnitudes of current row ${ }_{i}$ and column ${ }_{i}$ elements, and $2^{-t}$ is a machine precision ( $t$ bits in mantissa).

For the first order $I D$, the matrix $P$ has the same non-zero entry pattern as the original matrix. For a second order or higher $I D$, matrix $\mathrm{P}$ has one or more additional entries near the locations of the non-zero matrix entries, where the original matrix entries are zeros.

The incomplete decomposition order is selected automatically to ensure a stable and fast convergence, typical values for ID are 2 to 6 . Compared to other 3D commercial device simulators, NanoTCAD solver uses dramatically less memory (by a factor of 20), and CPU time is much smaller (by a factor of 10 to 20) for the similar problem and similar mesh size. Very large problems are solved within days instead of months compared to other commercial software. 
NanoTCAD simulator can solve a transient 3D multi-branched ion strike problem with 100,000 node unstructured mesh within 512MB memory. It can solve the problem with 3D unstructured mesh for up to 500,000 nodes within 2GB memory.

This efficient linear solver made possible to use adaptive unstructured 3D mesh generation for a transient 3D multi-branched ion strike problem.

\section{Novel Multiscale Single-Event Radiation Effect Models}

We are developing multiscale single-event models, which better reflect the true space environment and help to:

(i) better understand and predict response of nano-devices and novel materials to the space radiation environment, particularly high atomic number and energy particles (HZE particles) and energetic protons, and the interaction with materials and devices;

(ii) develop and assess radiation hardening techniques for space electronics;

(iii) better evaluate the radiation response of new electronic devices and circuits at early design stage;

(iv) reduce the amount radiation-laboratory testing, hence reducing the cost and time of exploration electronic system design.

These new models have required development of a new 3D adaptive mesh generation tool (which we have named Germes, for Gerris-based Mesher) to ensure better accuracy of the simulations and more reliable prediction of radiation issues in nanoscale technologies and devices.

The adaptive 3D mesh is particularly important for accurate modeling of complex, multi-branched tracks of single events (ion strikes) computed by MRED/Geant4 tools from Vanderbilt University, like, for example, presented in Fig.4 .

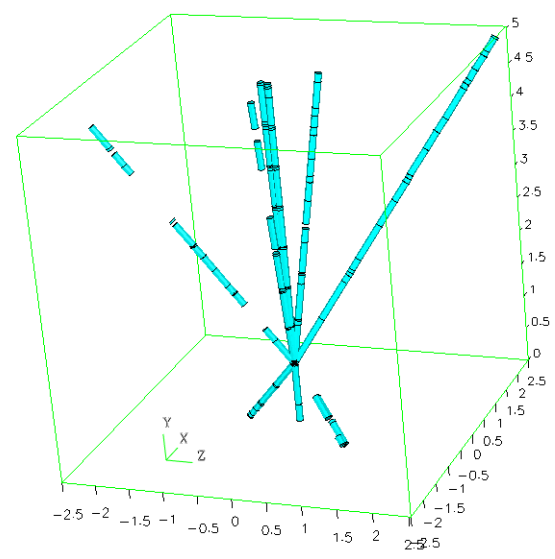

Fig. 4. View of "event 267" track-energy data ("tsv" file), plotted using "filaments" in CFDRC 3D software package. Boundaries of track segments from Geant4 are visible as black stripes. The incident particle was moving in the $+Y$ direction (from bottom to top). All sizes are in $\mu \mathrm{m}$. 
The NanoTCAD package contains an unstructured mesh solver. This makes a wider choice of possible meshes and refinement methods. Ones of particular interest are binary-tree (bin-tree) mesh-refinement methods. The mesh refinement is based on bin-tree levels, to discretize each particular part of the sub-domain. In this case, one can generate the refined grid cells/points in the volume of the transistor in the vicinity of particle tracks. We use more levels for higher energy deposition in the vicinity of tracks, Fig. 5.

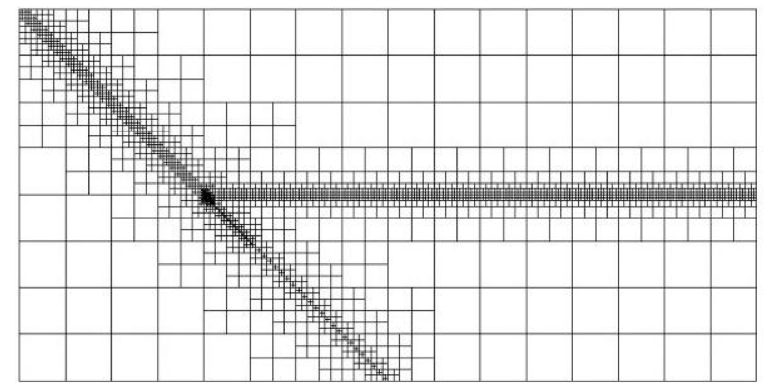

(a)

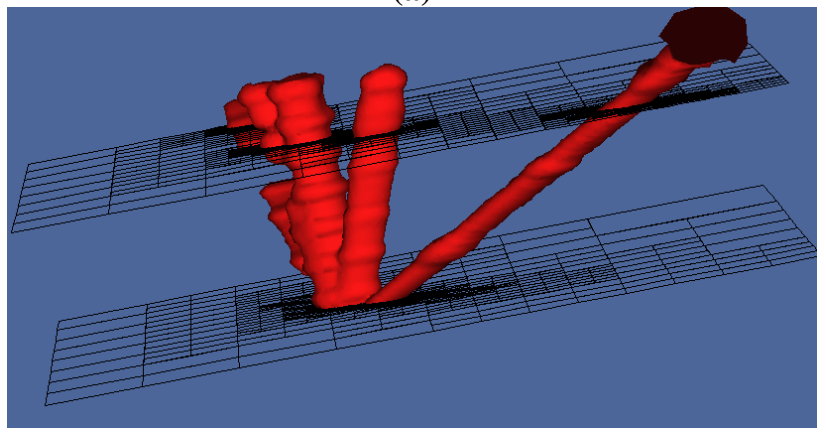

(b)

Fig. 5. (a) Adaptive mesh generation for $2 \mathrm{D}$ example case, which uses more levels for higher energy deposition in the vicinity of tracks, and (b) for the real 3D event 267 tracks. Different isosurface of energy value is plotted with more details. The adaptive mesh refinement in the vicinity of tracks is shown in different plane cross-sections at $\mathrm{Y}=\mathrm{const}$.

The advantage of this approach is that the refined mesh is quite localized in the vicinity of tracks only, dramatically saving the computational time. The difficulty of this approach is a much more complicated implementation. For our experiments, we have used the open-source modules from Gerris flow solver [6], [7]. It has 2D and 3D capabilities, and both static and dynamic mesh refinement modules.

We have developed the interfaces from MRED/GEant4 radiation event data to the Gerris mesh generation module and performed a series of tests for the mesh refinement with different particle-track models in the volume of the devices, both 2D and 3D cases. Fig. 5 shows bin-tree mesh refinement examples in the vicinity of ion tracks 
for $2 \mathrm{D}$ and $3 \mathrm{D}$ test cases. One can see that the mesh refinement is local, in the vicinity of tracks only. This is very important for the efficiency of computations for very large problems .

\section{Nanotcad Transient Simulations with MRED/Geant4 Tracks with Developed Accurate Radiation Event Model}

The 3D mesh, created using the new CFDRC tools described above, can be used for transient 3D simulations of semiconductor nano-device response to such a radiation event generated by the Vanderbilt MRED/Geant4 interface. First such simulations have been performed at CFDRC, for a NMOS-transistor with external circuit (Fig. 6).

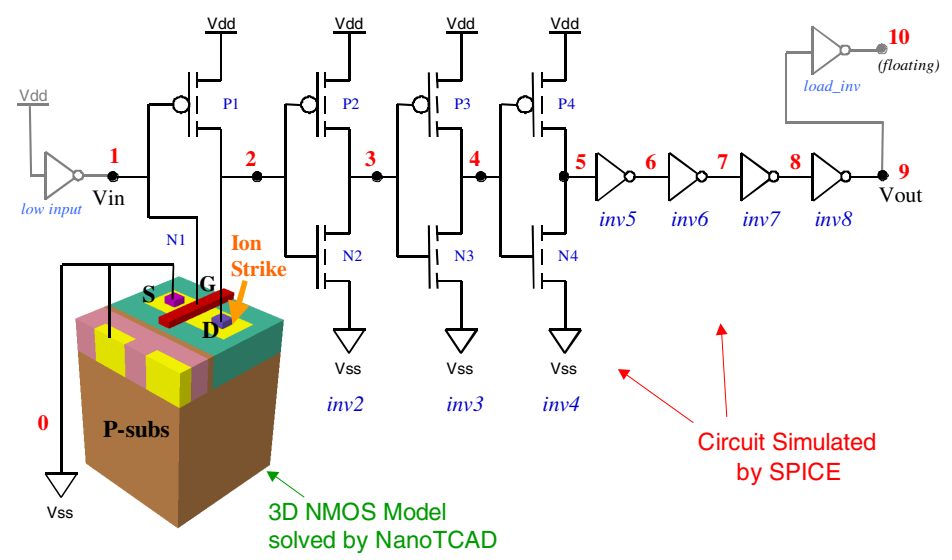

Fig. 6. The 8-inverter chain simulation setup used for our mixed-mode simulations

The ion strike duration time-scale is one ps, but the time-scale of transient device respond is up to six orders of magnitude higher, $1 \mu \mathrm{s}$. Numerical simulation of such transient behavior is difficult and required adaptive integration in time. Sample results are shown in Fig. 7 (b), the collected charge conservation is very good.

For the device response computation, we simulated the NMOS device using the CFDRC NanoTCAD 3D solver [6] and the rest of the circuit in Spice (using the CFDRC mixed-mode package, MixSpice). In the NMOS 3D device volume, we applied the radiation event data obtained from Vanderbilt MRED simulation, which were then used in CFDRC new tool, Germes, to generate a 3D model with adaptive meshing around the ion tracks. The multi-branched ion-track segments outside of the NMOS 3D TCAD model can be ignored, as not affecting the device behavior. A series of test simulations was done, with different mesh sizes, with adaptive mesh refined near the ion tracks and doping profiles. We found that a 3D mesh with about 30,000 nodes for NMOS was sufficiently good, producing results for currents and collected charge $<8 \%$ different from the results obtained for 3D mesh sizes of 80,000 

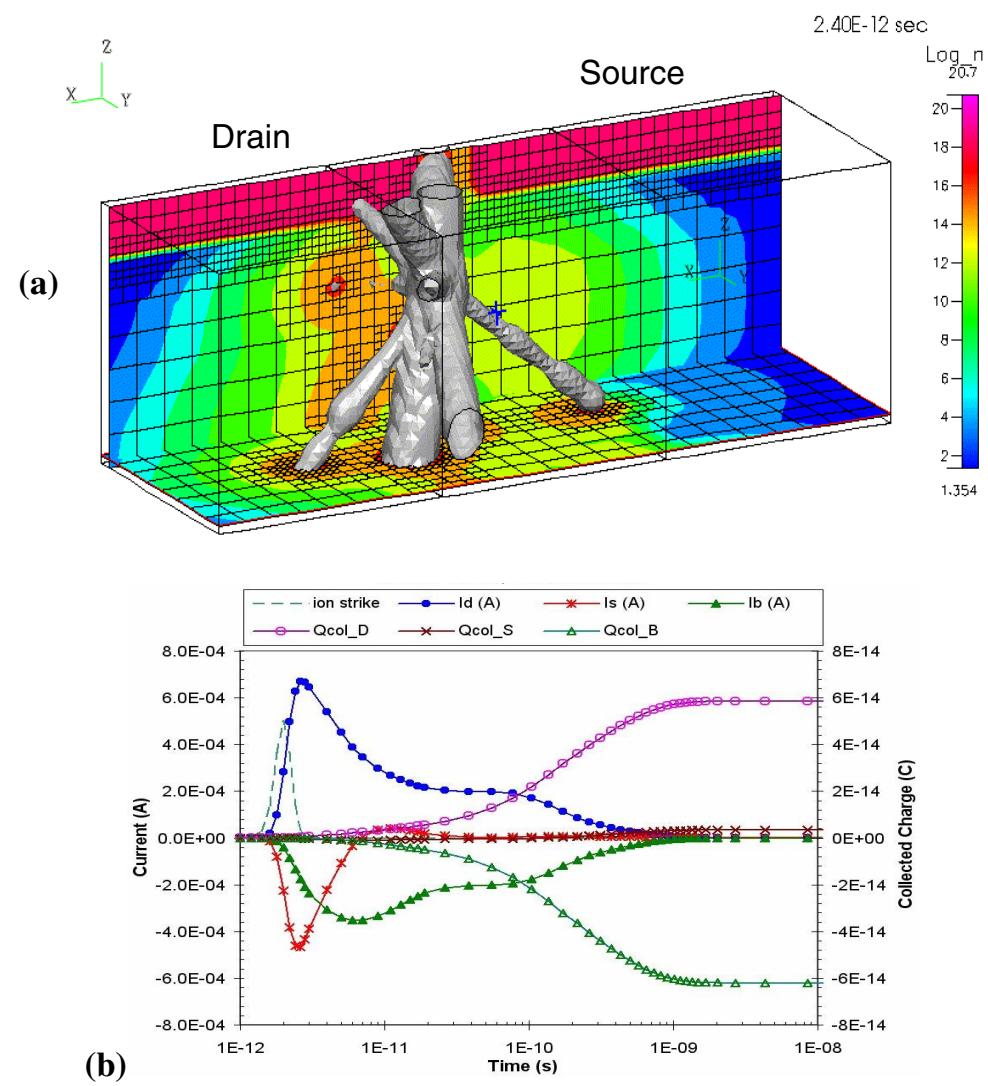

Fig. 7. Multiscale numerical simulations : MRED/Geant4 nuclear reaction radiation event 3D simulation in NMOS by CFDRC NanoTCAD. (a) Ion tracks (isosurface of deposited energy), $3 \mathrm{D}$ mesh locally refined around tracks, and resulting electron concentration (color map), at $\mathrm{t}=$ 2.4 ps. (b) Transient currents flowing through the contacts and the collected charges versus time (for this event, the total collected charge Qcol $=59 \mathrm{fC}$ ).

and 120,000 nodes, while saving the computation time dramatically. Our typical transient simulation with $3 \mathrm{D}$ mesh of $30 \mathrm{~K}-40 \mathrm{~K}$ nodes takes about $40-60$ minutes on a 2GHz CPU.

\section{Conclusions}

A description of multiscale numerical models involving fluid carrier transport and nuclear ion track generation, adaptive 3D meshing approach, and efficient solution method for large 3D problems using unstructured meshes has been provided. Multiscale models and adaptive dynamic 3D mesh generation presented in this paper enable efficient simulations of transient semiconductor device response to realistic multibranched track, produced by ionizing particle. The developed approach efficiently 
uses computer resources, makes possible solution of transient 3D multi-branched ion strike problems on a regular desktop computer.

Key features include an interface and adaptive meshing to allow simulations of single event radiation effects with nuclear reactions and secondary particles computed by MRED/Geant4 tools from Vanderbilt University. As shown by many recent papers, the contribution of nuclear reactions to single event upset cross-section of modern ICs may be very significant.

\section{References}

1. Weller, R.A., Sternberg, A.L., Massengill, L.W., Schrimpf, R.D., Fleetwood, D.M.: Evaluating average and atypical response in radiation effects simulations. IEEE Trans. Nucl. Sci. 50, 2265-2271 (2003)

2. Kobayashi, A.S., Sternberg, A.L., Massengill, L.W., Schrimpf, R.D., Weller, R.A.: Spatial and Temporal Characteristics of Energy Deposition by Protons and Alpha Particles in Silicon. IEEE Trans. Nucl. Sci. 51, 3312 (2004)

3. Turowski, M., Raman, A., Schrimpf, R.: Non-Uniform Total-Dose-Induced Charge Distribution in Shallow-Trench Isolation Oxides. IEEE Trans. on Nuclear Science 51, 3166-3171 (2004)

4. CFDRC, NanoTCAD (2006), http://www.cfdrc.com/bizareas/microelec/micro_nano/

5. Fedoseyev, A.I., Turowski, M., Wartak, M.S.: Kinetic and Quantum Models for Nanoelectronic and Optoelectronic Device Simulation. J. of Nanoelectronics and optoelectronics 2, 234-256 (2007)

6. Popinet, S.: Numerical modelling of fluid flow. Marsden Update (July 2004)

7. Popinet, S.: Free Computational Fluid Dynamics. ClusterWorld, 2 (June 2004)

8. Fedoseyev, A.I., Bessonov, O.A.: Iterative solution for large linear systems for unstructured meshes with preconditioning by high order incomplete decomposition. Comput. Fluid Dynamics Journal 10, 296-304 (2001)

9. Kershaw, D.S.: The incomplete Cholesky-conjugate gradient method for the iterative solution of systems of linear equations. J. Comput. Phys. 2, 43 (1978) 